\title{
An adolescent case of limbic encephalitis with anti leucine- rich glioma inactivated 1 antibodies
}

\author{
Małgorzata Janas-Kozik', Marek Krzystanek², Lena Cichoń1, Ireneusz Jelonek' ${ }^{1}$ Andrzej Siwiec ${ }^{1}$, Krzysztof \\ Krysta $^{2,+}$, Janusz K Rybakowski
}

\begin{abstract}
Background: Limbic encephalitis associated with leucine-rich glioma inactivated 1 (LGI1) antibodies can initially have either neurological or psychiatric manifestation.

Subject and methods: The paper describes a case of a 14-year old girl with LGI1 antibodiesassociated limbic encephalitis with initial symptoms suggesting psychotic disturbances.

Results: The magnetic resonance imaging (MRI) of the head was normal and the electroencephalography (EEG) presented low voltage with inter-hemispheric asymmetry and paroxysmal series of delta and theta waves. Blood antibody tests detected the presence of the LGI1 antibodies. Apart from psychotic symptoms, the patient also presented epileptic seizures, instability of the autonomous nervous system, dysphagia and catatonia. Initial treatment with antipsychotics resulted in the neuroleptic malignant syndrome (NMS). Significant improvement of the patient's condition appeared upon the administration of immunoglobulins and methylprednisolone.
\end{abstract}

Conclusions: Diagnosis and treatment of the limbic encephalitis in the young patient may be difficult due to a variety of reasons. A determination of anti-LGI1 antibodies also in adolescents presenting psychotic symptoms may be considered.

\section{Keywords}

Limbic encephalitis, Psychosis, Leucine-rich glioma inactivated 1 antibodies

\section{Introduction}

Leucine-rich glioma inactivated 1 (LGI1) proteins 1-4 play an important role in synaptic transmission and myelination, and LGI1 is one of the novel autoantigens in autoimmune encephalitis [1]. The LGI1 gene is located on chromosome 10 and its mutation causes autosomal dominant lateral temporal epilepsy [2]. A subgroup of patients with the LGI1 autoantibodies is diagnosed as faciobrachial dystonic seizures (FBDS), presenting very frequent brief stereotyped contractions of face and arm [3]. In this condition, spectacular effects have been obtained with immunotherapy [4].
The LGI1-associated encephalitis is the most frequent form of autoimmune encephalitis in adults. The median age of patients with LGI1 antibody-associated limbic encephalitis is 60 years, and most do not suffer from cancer. The symptoms are diverse, with a severe shortterm memory loss, psychiatric disturbances and various types of seizures, including FBDS [5]. The condition may be complicated with severe hyponatremia in up to $60 \%$ of cases [6]. The majority of the patients improve with corticosteroids, plasma exchange, or intravenous immunoglobulin [5].

\footnotetext{
'Developmental Age Clinical Ward, Department of Psychiatry and Psychotherapy, Medical University of Silesia, John Paul II Pediatric Center, Gabrieli Zapolskiej 3; 41-218 Sosnowiec, Poland

${ }^{2}$ Department of Rehabilitation Psychiatry, Medical University of Silesia, Ziołowa 45/47, 40-635 Katowice, Poland

32Department of Adult Psychiatry, Poznan University of Medical Sciences, Szpitalna 27/33, 60-572 Poznań, Poland

${ }^{\dagger}$ Author for correspondence: Krzysztof Krysta, PhD, Department of Rehabilitation Psychiatry, Medical University of Silesia, Ziołowa 45/47, 40-635 Katowice, Poland, Phone/Fax: 0048322059260, email: krysta@mp.pl
} 
The most cases of autoimmune limbic encephalitis in children occur with the antibodies against $\mathrm{N}$-methyl-D-aspartate (NMDA) glutamatergic receptor [7]. However, the limbic encephalitis is fairly rare in this population. In a recent multicenter European study Haberlandt et al. [8] showed only 10 cases of limbic encephalitis in patients below 18 years of age. Also, two recent case reports describing LGI1-associated limbic encephalitis pertain to adults: the first-a 41-year old female, with confusional state and cranial magnetic resonance imaging (MRI) abnormality [9], and, the second - a 62-year old male, with presentation of seizures, multiple mental symptoms and memory loss [10].

\section{Aim of the study}

In this paper, we report a case of a 14-year old girl initially presenting psychotic symptoms and diagnosed with limbic encephalitis with antiLGI1 antibodies.

\section{Case report}

An adolescent female patient, aged 14, without personal or family history of any psychiatric and neurologic disorders, was consulted on an urgent basis due to psychomotor agitation and aggressive behavior. A week earlier, an epileptic incident had occurred, manifested with lockjaw, sialorrhea, jerking of the left lower limb, and the loss of contact with the environment. Additional symptoms reported by the parents during an objective interview included recurrent headaches. The MRI of the head ruled out the proliferative process in the central nervous system, and in the electroencephalography (EEG), the paroxysmal changes and seizures was not present. The consulting physician observed difficult verbal contact due to the dissociation of thinking, disorientation in place and in personal data, irritable mood, inappropriate affect and psychomotor agitation. Moreover, the girl expressed delusions of reference and persecution with delusional identification of persons. The neurological examination excluded any neurological syndrome.

The patient was diagnosed as a psychotic episode and was admitted to the Developmental Age Psychiatry and Psychotherapy Clinical Ward. Computed tomography (CT) of the head revealed no changes. The lumbar puncture showed no abnormalities. Cerebrospinal fluid (CSF) culture and polymerase chain reaction (PCR) against viruses Herpes simplex type $I$ and II, Borrelia burgdorferi and Mycobacteria tuberculosis were negative. According to the presented symptoms, the treatment with aciclovir (max. $1500 \mathrm{mg} / \mathrm{d}$ ), haloperidol (max. $9 \mathrm{mg} / \mathrm{d}$ ) and risperidone (max. $3 \mathrm{mg} / \mathrm{d}$ ) was initiated.

On the $3^{\text {rd }}$ day of hospitalization, the unstable arterial blood pressure (ABP), increased heart rate, the increase of creatinine kinase $(\mathrm{CK})$, leukocytosis and increased body temperature $\left(38^{\circ} \mathrm{C}\right)$ were observed. Because of the suspected neuroleptic malignant syndrome (NMS) both neuroleptics were immediately withdrawn. After stabilization of the clinical state, on the $5^{\text {th }}$ day of hospitalization, olanzapine (max. $15 \mathrm{mg} / \mathrm{d}$ ) was gradually introduced. On the $16^{\text {th }}$ day of hospitalization the symptoms of NMS recurred, accompanied with stupor, rigidity, dysphagia, mutism and negativism. Due to the worsening of the somatic and mental state, olanzapine was also discontinued. The patient was moved to Intensive Care Unit (ICU).

After 7 days at the ICU, the clinical picture presented by the patient suggested a neurological disorder. Further diagnostics was continued at the Pediatrics Neurology (the $23^{\text {rd }}$ day of hospitalization). Regardless of no change in neuroimaging (MRI), EEG revealed a lowvoltage record with interhemispheric asymmetry and paroxysmal series of delta and theta waves. Differential diagnosis with the use of tandem mass spectrometry excluded aminoacidopathy, organic acidurias, fatty acid oxidation disorders, and certain types of urea cycle disorders. Another lumbar puncture revealed the presence of oligoclonal bands of IgG but with the correct IgG concentration and increased $\operatorname{IgG}$ de novo production index.

On the $26^{\text {th }}$ day of hospitalization (when the neurological causes of the symptoms were excluded) the patient was re-admitted to the psychiatric ward. The diagnostic procedure was continued at the psychiatric ward. All tests against tumor markers (beta HCG, AFP, CEA, Ca 125) were negative, CT of the chest and neck as well as ultrasonography (USG) of abdomen showed no abnormalities. fT4 was increased. TSH, fT3 and antithyroid antibodies were correct, which excluded a possibility of a diagnosis of Hashimoto's encephalopathy. Serum test against subacute sclerosing panencephalitis (SSPE) showed negative IgM and positive IgG anti-measles antibodies. The test was confirmed with an enzyme-linked immunosorbent assay 
(ELISA)-the result indicated a past infection, or a post-vaccinal reaction. The subsequent EEG repeated the previous findings. Further diagnostics against autoimmune disease showed the positive result for anti-nuclear antibodies (ANA). After one month of hospitalization, due to the worsening of the clinical state, new treatment with groprinosine, valproic acid, buspirone, methylprednisolone hemisuccinate, at a daily dose of $1 \mathrm{~g}$ for 5 days, and intravenous immunoglobulins (Intraglobin F), at a daily dose of $20 \mathrm{~g}$ for 5 days, was introduced. Despite the exacerbation of epileptic seizures, the treatment with immunoglobulins was continued.

The serum was sent to be tested against the NMDA receptor antibodies and the presence of antibodies against NMDA receptor in blood at the low-positive level was confirmed. The serum was tested against other antibodies characteristic for autoimmune encephalitis and the presence of anti-LGI-1 antibodies was detected. The treatment was continued in the Clinic of Developmental Age Neurology.

After three months of hospitalization the patient was discharged from hospital in a good general condition. The girl became conscious and active with only periodically disturbed orientation and a slight prolonged reaction. Mild sensory aphasia and labile affect with retrograde amnesia were still present. She reported no hallucinations and expressed no delusions (Figure 1).

\section{Discussion}

To the best of our knowledge, this is the first case in Poland and one of a very few cases in the world of children diagnosed with the limbic encephalitis with anti-LGI1 antibodies. The most conspicuous finding was that the illness started with acute psychotic symptoms which caused admission to a psychiatric ward. Both during the onset and subsequent course of illness other clinical symptoms characteristic of limbic encephalitis with anti-LGI1 antibodies were present, such as disorientation, disturbances of consciousness, seizures, irritable mood, inappropriate affect and psychomotor agitation as well as vegetative system disturbancessweating and sialorrhea [11]. The diagnosis of autoimmune limbic encephalitis in the patient was done precisely on the basis of clinical picture, and was additionally confirmed with the analysis of characteristic of anti-LGI1 antibodies levels [9]. Also an infection as a cause of limbic encephalitis was excluded. The MRI, EEG and
CSF analyses proved to be helpful in the course of the diagnosis. In addition, tumor markers assessment and imaging were performed to rule out a paraneoplastic form of autoimmune limbic encephalitis [11].

A differential diagnosis of this case could include other psychotic disturbances including schizophrenia. The mere detecting of antiNMDA antibodies is not sufficient for the diagnosis since such antibodies may be present in a proportion of patients with the first episode of psychosis [12]. Psychotic symptoms in the clinical picture of limbic encephalitis refer to the glutamatergic theory of schizophrenia. In this theory, an insufficiency of NMDA receptors is postulated as a basis for psychotic symptoms [13]. However, recently, an interaction between LGI1 protein and glutamatergic system was also demonstrated [14].

An association between LGI1 protein disturbances and abnormal seizure activity has been found in clinical and genetic studies $[2,3]$. This was also reflected in our patient by clinical symptoms and EEG findings. An epileptic incident had preceded the onset of psychosis and epileptic seizures occurred during the course of illness. Although initial EEG recording was normal, during subsequent recordings a highly pathological picture was obtained: low voltage with inter-hemispheric asymmetry and paroxysmal series of delta and theta waves.

The case illustrates initial difficulties in the treatment of psychiatric symptoms. The administration of antipsychotic drugs resulted in the emergence of NMS after administering drugs with a high anti-dopaminergic activity (risperidone, haloperidol), and also the recurrence of NMS after olanzapine was thought to have lowered this activity. The therapeutic effect in this limbic encephalitis was obtained with immunotherapy (immunoglobulins) and methylprednisolone. Good results of this therapeutic procedure in LGI1 antibodiesassociated conditions have been reported in other studies $[4,5,10]$. When methylprednisolone and immunoglobulins or plasmapheresis do not bring the desired therapeutic effect, it is recommended to include pharmacotherapy of the second linerituximab or cyclophosphamide [15].

\section{Conclusions}

Our report underlines diagnostic and therapeutic difficulties when the first psychotic episode 


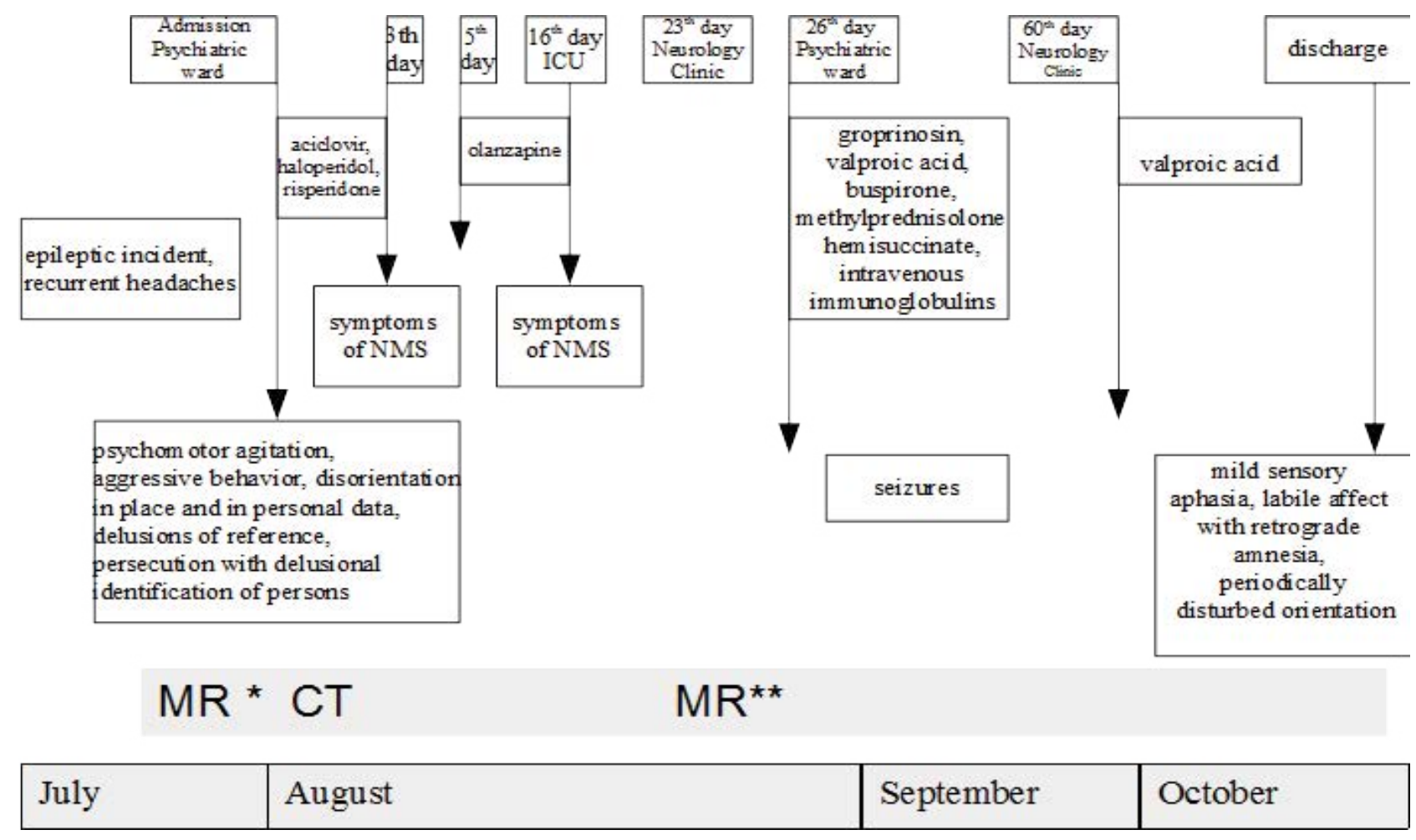

Figure 1: Disease course and management.

is seen in an adolescent. Many factors may contribute to the delay of proper diagnosis, and consequently, to adequate therapy of the limbic encephalitis in such a patient. Therefore, during the diagnostic process in children with the first psychotic episode, suspected with encephalitis, testing for the anti-LGI1 antibodies may also be considered.

\section{Acknowledgments}

The authors thank Professor Angela Vincent (Oxford) for her clinical consultation and support in the diagnostic process, described in the paper.

\section{Conflict of interest}

The authors declare no conflict of interest.

\section{References}

1. Kegel L, Aunin E, Meijer D, et al. LGI proteins in the nervous system. ASN. neuro 5(3), 167181 (2013).

2. Manna I, Mumoli L, Labate A, et al. Autosomal dominant lateral temporal epilepsy (ADLTE): absence of chromosomal rearrangements in LGI1 gene. Epilepsy. Res 108(3), 597-599 (2014).

3. Irani SR, Michell AW, Lang B, et al. Faciobrachial dystonic seizures precede Lgi1 antibody limbic encephalitis. Ann. Neurol 69(5), 892-900 (2011).

4. Irani SR, Stagg CJ, Schott JM, et al. Faciobrachial dystonic seizures: the influence of immunotherapy on seizure control and prevention of cognitive impairment in a broadening phenotype. Brain 136(Pt 10), 3151-3162 (2013).

5. Lai M, Huijbers MG, Lancaster E, et al. Investigation of LGI1 as the antigen in limbic encephalitis previously attributed to potassium channels: a case series. Lancet. Neurol 9(8), 776-785 (2010)

6. McQuillan RF, Bargman JM. Hyponatraemia caused by LGI1-associated limbic encephalitis. NDT. Plus 4(6), 424-426 (2011).

7. Armangue T, Petit-Pedrol M, Dalmau J. Autoimmune encephalitis in children. $J$. Child. Neurol 27(11), 1460-1469 (2012).

8. Haberlandt E, Bast T, Ebner A, et al. Limbic encephalitis in children and adolescents. Arch. Dis. Child 96(2), 186-191 (2011).

9. Shen $\mathrm{N}$, Ren $\mathrm{H}, \mathrm{Wu}$ J, et al. A rare case of limbic encephalitis with anti leucine-rich glioma inactivated-1 (LGI1) antibodies. Neuro. Endocrinol. Lett 35(2), 95-97 (2014).

10. Mayasi Y, Takhtani D, Garg N. Leucine-rich glioma-inactivated protein 1 antibody encephalitis: A case report. Neurol. Neuroimmunol. Neuroinflamm 1(4), e51 (2014).
11. Kayser MS, Kohler CG, Dalmau J. Psychiatric manifestations of paraneoplastic disorders. Am. J. Psychiatry 167(9), 1039-1050 (2010).

12. Pathmanandavel K, Starling J, Merheb V, et al. Antibodies to surface dopamine-2 receptor and $\mathrm{N}$-methyl-D-aspartate receptor in the first episode of acute psychosis in children. Biol. Psychiatry 77(6), 537-547 (2015).

13. Olney JW, Newcomer JW, Farber NB. NMDA receptor hypofunction model of schizophrenia. J. Psychiatr. Res 33(6), 523533 (1999).

14. Boillot M, Huneau C, Marsan E, et al. Glutamatergic neuron-targeted loss of LGI1 epilepsy gene results in seizures. Brain 137(Pt 11), 2984-2996 (2014).

15. Sonn TS, Merritt DF. Anti-NMDA-Receptor Encephalitis: An Adolescent with Ovarian Teratoma. J. Pediatr. Adolesc. Gynecol 23(1), e141-144 (2010) 